\title{
Diabetic ketoacidosis: a challenging diabetes phenotype
}

\author{
Cliona Small, Aoife M Egan, El Muntasir Elhadi, Michael W O’Reilly, \\ Aine Cunningham and Francis M Finucane \\ HRB Clinical Research Facility, Galway University Hospitals, National University of Ireland, \\ Galway, Ireland
}

\author{
Correspondence \\ should be addressed \\ to F M Finucane \\ Email \\ francis.finucane@hse.ie
}

\section{Summary}

We describe three patients presenting with diabetic ketoacidosis secondary to ketosis prone type 2, rather than type 1 diabetes. All patients were treated according to a standard DKA protocol, but were subsequently able to come off insulin therapy while maintaining good glycaemic control. Ketosis-prone type 2 diabetes (KPD) presenting with DKA has not been described previously in Irish patients. The absence of islet autoimmunity and evidence of endogenous beta cell function after resolution of DKA are well-established markers of KPD, but are not readily available in the acute setting. Although not emphasised in any current guidelines, we have found that a strong family history of type 2 diabetes and the presence of cutaneous markers of insulin resistance are strongly suggestive of KPD. These could be emphasised in future clinical practice guidelines.

\section{Learning points:}

- Even in white patients, DKA is not synonymous with type 1 diabetes and autoimmune beta cell failure. KPD needs to be considered in all patients presenting with DKA, even though it will not influence their initial treatment.

- Aside from markers of endogenous beta cell function and islet autoimmunity, which in any case are unlikely to be immediately available to clinicians, consideration of family history of type 2 diabetes and cutaneous markers of insulin resistance might help to identify those with KPD and are more readily apparent in the acute setting, though not emphasised in guidelines.

- Consideration of KPD should never alter the management of the acute severe metabolic derangement of DKA, and phasing out of insulin therapy requires frequent attendance and meticulous and cautious surveillance by a team of experienced diabetes care providers.

\section{Background}

The occurrence of hyperglycaemic ketosis and diabetic ketoacidosis (DKA) as manifestations of type 2 rather than type 1 diabetes has been well established for some time $(1,2)$. However, awareness of this among clinicians is variable, with a widely held misconception that ketosis is synonymous with absolute rather than relative insulin deficiency and with type 1 diabetes. Many guidelines on the management of DKA do not highlight the possibility of 'ketosis-prone diabetes' (KPD) $(3,4)$, though some do
$(5,6)$. Heterogeneity exists in the approach to diagnosing KPD. Some authorities emphasise adiposity and ethnicity (7), whereas others consider beta-cell function and markers of beta-cell autoimmunity in assessing the likelihood of KPD (8). However, applying these criteria in clinical practice can be challenging and other factors such as family history and the presence of cutaneous manifestations of insulin resistance such as skin tags or acanthosis nigricans might help to refine the diabetes 
phenotype and differentiate between type 1 and ketosis prone type 2 diabetes variants. This would have important implications for optimising therapy for individual patients who may avoid unnecessary indefinite insulin therapy. We sought to identify areas of uncertainty and potentially informative clinical features in the assessment and treatment of patients presenting with diabetic ketoacidosis.

\section{Case presentation}

Case 1

A 44-year-old Sudanese male presented to our emergency department with an episode of collapse, associated with a two-week history of severe thirst, polyuria, nocturia, lethargy and $10 \mathrm{~kg}$ weight loss. He had no history of note, was on no medications, had no allergies and never drank alcohol. He was drowsy with slurred speech but no focal neurological deficits. He was severely dehydrated with dry mucus membranes, reduced skin turgidity, tachycardia and hypotension. As shown in Table 1 , he had severe hyperglycaemia, ketonaemia and metabolic acidosis with low $\mathrm{pH}$ and bicarbonate and high lactate levels as well as severe hypernatraemia and hyperosmolar state. He had acute kidney injury secondary to rhabdomyolysis (creatine kinase $45000 \mu / \mathrm{L}$, normal <308). He was intubated and ventilated and transferred to the intensive care unit. He was managed according to our DKA/HHS protocol with aggressive intravenous fluid resuscitation and cautious normalisation of his sodium levels. He required six units per hour of intravenous insulin to restore euglycaemia over the initial $12 \mathrm{~h}$. On day two, he required continuous veno-venous haemodiafiltration for three days with subsequent normalisation of renal function. Initially on this, his corrected sodium dropped from 157 to 143 over four hours, so a hypertonic saline infusion was used for $48 \mathrm{~h}$ to avoid osmotic demyelination syndrome from over-rapid correction of hypernatraemia. He improved and was changed to a multiple daily injection (MDI) regime of eight units of insulin aspart with meals and 20 units of insulin detemir at night. There was no family history of diabetes, nor were there any skin tags or acanthosis nigricans, though the patient had a centripetal fat distribution on examination. He reported a high intake of sugar-sweetened beverages in the weeks prior to admission. Notwithstanding his presentation with diabetic ketoacidosis, his weight loss and the absence of a family history of diabetes or cutaneous markers of insulin resistance, his elevated BMI and central fat distribution, his ethnicity and the severity of his hyperosmolar state suggested a possible underlying type 2 diabetes phenotype. We discharged him on MDI insulin and started metformin therapy six weeks after diagnosis, which led to a reduction in his insulin requirements to zero eight weeks after diagnosis and improvement in his HbA1c to $52 \mathrm{mmol} / \mathrm{mol}$. One month after diagnosis, his C-peptide levels suggested endogenous insulin secretion and intact beta-cell function. Thereafter, his anti-glutamic acid decarboxylase (GAD) and anti-Islet cell (IC2) antibodies were negative. Fourteen months after initial presentation,

Table 1 Anthropometric and metabolic characteristics of three patients at initial presentation with diabetic ketoacidosis.

\begin{tabular}{l}
\hline \\
\hline BMI (kg/m²) \\
Plasma glucose (mmol/L) \\
HBA1c (mmol/mol) \\
Plasma ketones (mmol/L) \\
Urine ketones (semi-quantitative) \\
pH (arterial) \\
pC02 (arterial) \\
HC03- (mmol/L) \\
Plasma lactate (mmol/L) \\
Plasma sodium (mmol/L) \\
Triglycerides (mmol/L) \\
eGFR (mL/min) \\
Serum osmolality (mmol/kg) \\
Anti-GAD antibodies (U/mL) \\
Anti-islet cell (IC2) antibodies (U/mL) \\
C-peptide (pmol/L)
\end{tabular}

*One month after the initial presentation. **One week after initial presentation. BMI, body mass index; eGFR: estimated glomerular filtration rate; GAD, glutamic acid decarboxylase.

\begin{tabular}{c}
\hline Patient 2 \\
\hline 44 \\
34.9 \\
142 \\
5 \\
$' 3+'$ \\
7 \\
1.21 \\
5.9 \\
2.0 \\
129 \\
1.1 \\
$>90$ \\
320 \\
Negative \\
Negative \\
$562 * *$
\end{tabular}

\begin{tabular}{c}
\hline Patient 3 \\
\hline 34.7 \\
30 \\
113 \\
$\mathrm{n} / \mathrm{a}$ \\
'3+' \\
7.31 \\
4.56 \\
16.9 \\
1.3 \\
138 \\
2.8 \\
$>90$ \\
311 \\
Negative \\
Negative \\
n/a
\end{tabular}


he continues to do well on metformin monotherapy with an $\mathrm{HbA} 1 \mathrm{c}$ in the non-diabetic range at $42 \mathrm{mmol} / \mathrm{mol}$.

\section{Case 2}

A previously well 45-year-old white Irish male farmer presented to the emergency department with a oneday history of abdominal pain and vomiting with associated dyspnoea, preceded by a four-week history of polyuria, polydipsia, lethargy and $9 \mathrm{~kg}$ weight loss. On examination, he appeared severely dehydrated, with hypotension, tachycardia and tachypnoea. He had a large para-umbilical hernia, which appeared strangulated and obstructed on an abdominal CT scan. Moreover, he had diabetic ketoacidosis as outlined in Table 1. His renal and liver function and lactate were normal. We considered the severity of his DKA was aggravated by his bowel obstruction, notwithstanding his normal lactate level. The standard protocol for managing DKA was initiated with fluid resuscitation, electrolyte repletion and intravenous insulin prior to laparoscopic mesh repair of his hernia. He required in excess of 100 units of insulin per day initially to restore euglycaemia. He had an elevated BMI, a centripetal fat distribution, axillary and cervical skin tags and acanthosis nigricans and a strong family history of T2DM, all consistent with an insulinresistant phenotype. He also had a suboptimal lipid profile and microalbuminuria at diagnosis. We established the patient on a twice-daily mixed insulin regime prior to discharge and noted a progressive reduction in insulin requirements with restoration of glycaemic control. We started metformin five weeks after his initial presentation and stopped insulin completely three weeks later, when his $\mathrm{HbA1c}$ had improved to $48 \mathrm{mmol} / \mathrm{mol}$. Anti-GAD and anti-IC2 antibodies were negative. Forty-one months after his initial presentation with DKA, he remains well controlled on metformin monotherapy with a HbA1c of $44 \mathrm{mmol} / \mathrm{mol}$.

\section{Case 3}

A 23-year-old male with trisomy 21 , of mixed white Irish (mother) and Phillipino (father) ethnicity was referred by their GP to the emergency department with a one-week history of thirst, polydipsia, polyuria, $4 \mathrm{~kg}$ weight loss and abdominal pain, nausea and vomiting for $24 \mathrm{~h}$. He was tachypnoeic and tachycardic with ketotic breath. His metabolic indices were consistent with DKA (Table 1). He was treated with intravenous fluids and insulin according to protocol. His liver blood tests were consistent with mild fatty infiltration. His BMI was elevated, and he had axillary acanthosis nigricans, suggesting an insulinresistant phenotype. Moreover, his mother had T2DM and his brother had developed type 2 diabetes at age 24 years. His lipid profile was suboptimal with elevated triglycerides and low HDL $(0.9 \mathrm{mmol} / \mathrm{L})$. Initially, he required large doses of insulin to achieve glycaemic control and was discharged on day three on twice-daily mixed insulin (Novomix 30) 60 units mane and 50 units tarde. He also started metformin $500 \mathrm{mg}$ bd prior to discharge. By six weeks, his insulin dose had come down to 42 units/day, and he was weaned off insulin completely by 12 weeks. Anti-GAD and anti-IC2 antibodies were subsequently negative. C-peptide levels were not measured. His HbA1c improved significantly such that two years after his initial presentation, it was normal at $37 \mathrm{mmol} / \mathrm{mol}$ on metformin $850 \mathrm{mg}$ bd. The acanthosis nigricans and high BMI at presentation as well as the strong family history suggested type 2 rather than type 1 diabetes, notwithstanding this man's age, ethnicity and presentation with DKA. The negative antibodies and normalisation of glucose control without an ongoing requirement for insulin were felt to confirm the diagnosis.

\section{Discussion}

These cases highlight the fact that DKA is not synonymous with type 1 diabetes and permanent, autoimmune destruction of beta cells, but may arise from reversible beta cell failure in patients with phenotypic features of type 2 diabetes. In our first patient, even though there was no family history of type 2 diabetes or cutaneous manifestations of insulin resistance, his ethnicity, centripetal fat distribution and degree of hyperosmolarity at presentation suggested an insulin-resistant phenotype. Our approach (as with any patient with DKA) was to initiate treatment with insulin acutely and to consider introducing an insulin sensitiser (metformin) and reducing the insulin dose only after glycaemic control was restored and the severe metabolic derangements had fully resolved. We did not rely on islet autoantibodies or C-peptide levels in making a provisional diagnosis of KPD. Rather, we were guided by clinical features apparent at the outset. We were cautious to supervise his insulin dose reduction and blood glucose monitoring closely, with twice-weekly telephone contact with the diabetes nurse and regular clinical assessment.

Our second patient was similar to the first in that he had osmotic symptoms in the weeks prior to presentation and a centripetal fat distribution. However, KPD has not 
previously been described in a patient of white Irish ethnicity, usually occurring in patients of black African or Asian descent (9). Notwithstanding his ethnicity, the prominence of his insulin-resistant phenotype with skin tags, acanthosis, a strong family history of type 2 diabetes and high insulin requirements as well as his dyslipidaemia and prevalent microalbuminuria at diagnosis mandated consideration of KPD. As in all three cases, treatment according to our DKA protocol was initiated acutely and the consideration of KPD only influenced management once the acute metabolic derangements had been reversed with fluid resuscitation and insulin.

Our third case was also atypical of a patient with newly diagnosed type 2 diabetes, given his relative youth. However, the presence of cutaneous markers of insulin resistance and a very strong family history of type 2 diabetes prompted us to consider KPD, after his acute metabolic derangements were managed with fluids and insulin. All three patients were negative for anti-GAD and anti-IC2 antibodies, but (as in the cases here) often these results are only available weeks after the initial presentation, at least in our institution, so do not currently inform our initial phenotypic assessment. Nonetheless, a heavy emphasis is placed on their utility in a number of proposed classification schemes for KPD, in an attempt to predict better which patients might ultimately be weaned off insulin therapy vs requiring it for life (8). Some also suggest BMI is an important consideration (7), but we regard it as a relatively crude and unhelpful marker of excess body fat or its distribution. The ' $\alpha \beta$ ' system (8), which considers beta-cell function (measured with C-peptide) as well as islet autoimmunity, seems the best to us although we had not routinely measured C-peptide in all potential KPD patients up to now and in our first two patients here, it was measured weeks after their initial presentation. Moreover, although international clinical guidelines have recently started to emphasise KPD as an important consideration in patients presenting with DKA, consensus on the optimal way to diagnose and manage $\mathrm{KPD}$ is lacking.

Our patients highlight the variability in terms of age, ethnicity and adiposity associated with ketosisprone type 2 diabetes. The mechanistic basis for absolute, albeit transient absolute beta-cell failure in KPD is not fully established but is thought to involve glucotoxicity secondary to subacute or chronic hyperglycaemia, probably aggravated by underlying insulin resistance (10). Although rarer causes of 'non-type 1' DKA such as HNF1A MODY (11) and euglycaemic DKA in patients on
SGLT2 inhibitors (12) have been described, KPD is the most likely alternative diagnosis to type 1 diabetes. In carefully reducing the insulin dose over weeks where KPD is suspected, we are adopting an approach of hoping for the best, anticipating the worst and taking what comes.

\section{Declaration of interest}

Francis Finucane has received honoraria, travel grants and has served on advisory boards for Novo Nordisk, Eli Lilly, Pfizer Inc., Sanofi-Aventis, Astra Zeneca, Merck-Serono, Boehringer Ingelheim, Janssen and Novartis. Aoife Egan has received honoraria and travel grants from Novo Nordisk, Eli Lilly, Pfizer Inc., Sanofi-Aventis, Astra Zeneca, Boehringer Ingelheim, Janssen and Novartis. Aine Cunningham, El Muntasir Elhadi, Michael W O'Reilly and Cliona Small have no conflicts of interest to declare.

\section{Funding}

This research did not receive any specific grant from any funding agency in the public, commercial or not-for-profit sector.

\section{Patient consent}

All patients provided written, signed, informed consent for their anonymised information to be included in this case series.

\section{Author contribution statement}

Each author contributed to the study design, description of the cases and data collection and analysis. Francis M Finucane was the treating physician for two patients and Michael W O'Reilly for the third.

\section{References}

1 Banerji MA, Chaiken RL, Huey H, Tuomi T, Norin AJ, Mackay IR, Rowley MJ, Zimmet PZ \& Lebovitz HE 1994 GAD antibody negative NIDDM in adult black subjects with diabetic ketoacidosis and increased frequency of human leukocyte antigen DR3 and DR4. Flatbush diabetes. Diabetes 43 741-745. (doi:10.2337/diab.43.6.741)

2 Umpierrez GE, Casals MM, Gebhart SP, Mixon PS, Clark WS \& Phillips LS 1995 Diabetic ketoacidosis in obese African-Americans. Diabetes 44 790-795. (doi:10.2337/diab.44.7.790)

3 Gosmanov AR, Gosmanova EO \& Dillard-Cannon E 2014 Management of adult diabetic ketoacidosis. Diabetes, Metabolic Syndrome and Obesity 7 255-264. (doi:10.2147/DMSO.S50516)

4 Joint British Diabetes Societies Inpatient Care Group 2013 Association of British Clinical Diabetologists. The Management of Diabetic Ketoacidosis in Adults, Second Edition. Updated September 2013. Accessed at http://www.diabetologists-abcd.org.uk/JBDS/JBDS.htm.

5 American Diabetes Association 2016 Standards of medical care in diabetes - 2016 7. Approaches to glycemic treatment. Diabetes Care 39 (Supplement 1) S52-S59. (doi:10.2337/dc16-s010)

6 Kitabchi AE, Umpierrez GE, Miles JM \& Fisher JN 2009 Hyperglycemic crises in adult patients with diabetes. Diabetes Care 32 1335-1343. (doi:10.2337/dc09-9032)

7 Umpierrez GE, Woo W, Hagopian WA, Isaacs SD, Palmer JP, Gaur LK, Nepom GT, Clark WS, Mixon PS \& Kitabchi AE 1999 Immunogenetic analysis suggests different pathogenesis for obese and lean African- 
Americans with diabetic ketoacidosis. Diabetes Care 22 1517-1523. (doi:10.2337/diacare.22.9.1517)

8 Balasubramanyam A, Garza G, Rodriguez L, Hampe CS, Gaur L, Lernmark A \& Maldonado MR 2006 Accuracy and predictive value of classification schemes for ketosis-prone diabetes. Diabetes Care 29 2575-2579. (doi:10.2337/dc06-0749)

9 Balasubramanyam A, Nalini R, Hampe CS \& Maldonado M 2008 Syndromes of ketosis-prone diabetes mellitus. Endocrine Reviews 29 292-302. (doi:10.1210/er.2007-0026)

10 Mauvais-Jarvis F, Sobngwi E, Porcher R, Riveline JP, Kevorkian JP, Vaisse C, Charpentier G, Guillausseau PJ, Vexiau P \& Gautier
JF 2004 Ketosis-prone type 2 diabetes in patients of sub-Saharan African origin: clinical pathophysiology and natural history of beta-cell dysfunction and insulin resistance. Diabetes 53 645-653. (doi:10.2337/diabetes.53.3.645)

11 Egan AM, Cunningham A, Jafar-Mohammadi B \& Dunne FP 2015 Diabetic ketoacidosis in the setting of HNF1A-maturity onset diabetes of the young. BMJ Case Reports 2015. (doi:10.1136/bcr-2014209163)

12 Taylor SI, Blau JE \& Rother KI 2015 SGLT2 inhibitors may predispose to ketoacidosis. Journal of Clinical Endocrinology and Metabolism 100 2849-2852. (doi:10.1210/jc.2015-1884)

Received in final form 15 December 2016

Accepted 31 January 2017 